\title{
The success of structural genomics
}

\author{
Thomas C. Terwilliger
}

Received: 1 June 2011/Accepted: 9 June 2011/Published online: 17 June 2011

(C) The Author(s) 2011. This article is published with open access at Springerlink.com

\begin{abstract}
The International Conference on Structural Genomics (ICSG 2011, http://www.icsg2011.org), held in Toronto Canada May 10-14, 2011 was a rich and exciting demonstration of how far structural genomics has come. Structural genomics has now matured into a field that includes both structure and the biology that structure enables. This has allowed targeting based on systematic approaches and on known biological importance and allows biochemical studies to be closely tied to structure determination. The wealth of purified proteins, clones, and chemical probes produced by structural genomics groups will enable a vast amount of follow-on research. The technologies, the structures, and the biology that were described at the meeting were at the cutting edge of science. Structural genomics has become a success.
\end{abstract}

Keywords Structural genomics - International Conference on Structural Genomics 2011 - International Structural Genomics Organization

The International Conference on Structural Genomics (ICSG 2011, http://www.icsg2011.org), held in Toronto Canada May 10-14, 2011 was a rich and exciting demonstration of how far structural genomics has come.

Structural genomics began a decade ago with the goal of discovering the 3-dimensional shapes of all macromolecules. The Agreed Principles of Structural Genomics had a broad and foundational vision (see http://www.isgo.org/m c/hinxton.html):

T. C. Terwilliger $(\bowtie)$

Los Alamos National Laboratory, Mail Stop M888, Los Alamos, NM 87545, USA

e-mail: terwilliger@lanl.gov
"This structural genomics initiative aims at the discovery, analysis and dissemination of three-dimensional structures of protein, RNA and other biological macromolecules representing the entire range of structural diversity found in nature. Such a complete knowledge will facilitate fundamental understanding and applications in biology, agriculture and medicine. The three-dimensional structures will be crucial for rational drug design, for advancing catalysis in chemistry and biotechnology, and for diagnosis and treatment of disease, as well as for advancing basic principles of biology."

Over the past decade structural genomics efforts worldwide have developed powerful new technologies, built high-throughput pipelines capable of determining hundreds of structures per year, determined thousands of structures, and combined structures with other biological information to build a stronger foundation for understanding biology.

Research teams and funding agencies in different parts of the world approached the overall goals of structural genomics with two rather different strategies. One strategy, taken by many in the US and in some efforts around the world, focused on covering as much of structure space as possible. The other strategy, taken in most of the rest of the world, focused on determining the structures that have the highest near-term importance first.

The broad scope and outstanding productivity shown by the talks at ICSG 2011 were one of the clearest indicators yet of the success of structural genomics. The talks showed the importance of both coverage-based and importancebased strategies for choosing targets and how merging these strategies and following up structure determination with biochemical experiments makes structural genomics even more powerful. 
Just a few examples from the many outstanding talks show the level of success in structural genomics. Wayne Hendrickson described the fantastic story of how their structural coverage-based selection of the channel protein TehA led to a structure of this protein from Haemophilus influenzae, how this led to prediction of the structure of the closely-related and highly important anion channel SLAC1 that regulates turgor pressure in plants, and how this provided the basis for a series of mutational and biochemical experiments to understand how the channel is controlled.

Merging the structural coverage and functional targeting approaches, Ray Stevens and Stefan Knapp showed how systematic structure determination focusing on structural coverage of GPCR proteins and of kinases can lead to many structures of exceptionally challenging and important targets and insights into their function.

Ted Baker described how a structural genomics approach to bacterial virulence genes showed how bacterial pili are rigidified by post-translational covalent linkages. Ian Wilson showed how the comprehensive structural coverage of $T$. maritima obtained from systematic targeting of structures from this genome has led to an integrated understanding of central metabolism in this organism.

It was clear from this meeting that structural genomics has now matured into a field that includes both structure and the biology that structure enables. This has allowed targeting based on systematic approaches and on known biological importance and allows biochemical studies to be closely tied to structure determination. The wealth of purified proteins, clones, and chemical probes produced by structural genomics groups will enable a vast amount of follow-on research. The technologies, the structures, and the biology that were described at the meeting were at the cutting edge of science. Structural genomics has become a success.

Acknowledgments This article was written jointly as an opinion piece for Instruct (www.structuralbiology.eu) and the Journal of Structural and Functional Genomics.

Open Access This article is distributed under the terms of the Creative Commons Attribution Noncommercial License which permits any noncommercial use, distribution, and reproduction in any medium, provided the original author(s) and source are credited. 